\title{
Educación de lo sensible: tras las huellas del pensamiento de Michel Foucault
}

//Education of the senses: in the footsteps

of Michel Foucault thought

\begin{tabular}{l|l} 
Investigaciones & $\begin{array}{l}\text { Revista Colombiana } \\
\text { de Educación, N. 63. } \\
\text { Segundo semestre de 2012, } \\
\text { Bogotá, Colombia. }\end{array}$
\end{tabular}

//Educação do sensível: os passos do

pensamento de Michel Foucault

Evelyn Dariana Marín Ramírez**

Recibido:30/06/2012

Evaluado:06/10/2012

\begin{abstract}
Hace parte del proyecto de investigación: "Cartografías de lo sensible". Trabajo de la Maestría en Motricidad y Desarrollo Humano del Instituto Universitario de Educación Física de la Universidad de Antioquia, 2011

Licenciada en Educación Física y estudiante de la Maestría en Motricidad y Desarrollo Humano en la línea Educación Corporal del Instituto Universitario de Educación Física, Universidad de Antioquia, Docente de la Universidad San Buenaventura, Medellín, Colombia. Grupo de Investigación: Estudios en Educación Corporal. Correo electrónico: darianam113@gmail.com
\end{abstract}

\section{Resumen}

El foco de atención de este escrito está puesto en el pensamiento de Michel Foucault, con la intención de re-correr sus conceptos y sus ideas como una ayuda para problematizar la educación de lo sensible, asunto que surge de la inconformidad con lo establecido por la hegemonía del pensamiento racionalista y que nos lleva a movilizar nuestro pensamiento en aras de decir "otras cosas" de la educación. Específicamente, en el pensamiento foucaultiano encontramos la relación del arte con la vida y lo leemos desde una perspectiva educativa, pretendiendo de este modo pensar la educación como "una de esas criaturas de Bacon, magma de mutilaciones y posibilidades larvarias, gritando bajo las intensidades insoportables de un renacimiento y que ha perdido su antiguo rostro, comenzando a darse uno nuevo, un nuevo cuerpo y una nueva aventura" (Deleuze, 1986, p. 16).

\section{Abstract}

The focus of this paper is placed on the thought of Michel Foucault, with the aim of re-run their concepts and ideas as an aid to problematize the Education of the senses, an issue that arises from dissatisfaction with the provisions of the hegemony of rationalist thought and leads us to mobilize our thinking in order to say other things about the education. Specifically, in the Foucaultian thinking, we find the relationship between art and life and read from an educational perspective, tryingth us to think of education as "one of those creatures of Bacon, magma and potential larval mutilation, screaming low intensities unbearable for a revival and has lost his old face, now learning a new one, a new body and a new adventure" (Deleuze, 1986, p. 16).

\section{Resumo}

O foco deste artigo é o pensamento de Michel Foucault, a fim de explorar seus conceitos e idéias como um auxílio para problematizar a educação do sensível, questão que surge da insatisfação com as disposições hegemônicas do racionalismo e que nos leva a mobilizar o nosso pensamento para dizer "outras coisas" sobre a educação. Especificamente, no pensamento foucaultiano encontramos a relação entre arte e vida, que lemos de uma perspectiva educacional, pretendendo assim, pensar a educação como "uma daquelas criaturas de Bacon, magma de mutilações e posibilidades larvárias, gritando sob intensidades insuportáveis de um renascimento e que perdeu seu rosto antigo, começando a dar-se um novo, um novo corpo e uma nova aventura "(Deleuze, 1986, p. 16).

\section{Palabras Clave}

Sensibilidad, educación, arte, vida, cuidado de sí, formación.

\section{Keywords}

Sensitivity, education, art life, self-care, training

\section{Palavras chave}

Sensibilidades, educação, arte, vida, cuidado de si, formação 


\title{
Introducción
}

\begin{abstract}
¿Por qué la fascinación? Ver supone la distancia, la decisión que separa, el poder de no estar en contacto y de evitar la confusión en el contacto. Ver significa, sin embargo, que esa separación se convirtió en encuentro. Pero ¿qué ocurre cuando lo que se ve, aunque sea a distancia, parece tocarnos por un contacto asombroso, cuando la manera de ver es una especie de toque, cuando ver es un contacto a distancia, cuando lo que es visto se impone a la mirada, como si la mirada estuviese tomada, tocada, puesta en contacto con la apariencia?
\end{abstract}

Maurice Blanchot

Una forma de construir un rizoma de una educación de lo sensible es, precisamente, poniendo en movimiento aquellos pensamientos de autores que sospechamos podemos leer y re-leer siempre con la fortuna de encontrar en ellos algo nuevo, pues en la tarea de problematizar la educación en perspectiva de lo sensible no queremos -como es pretensión de los racionalistas- apropiarnos de conocimientos infalibles, ideas cristalizadas y verdades absolutas; por el contrario, queremos establecer nuevas relaciones significativas que nos permitan poner, bajo diferentes textualidades, ideas, concepciones y pensamientos, no con el propósito de decir lo ya dicho, sino con la intención de problematizarlo en aras de movilizarlos, desterritorializarlos, generar resonancias y engendrar quizás nuevos pensamientos que nos permiten mudar de piel. Consideramos que siempre se queda algo escondido en los autores y cuando volvemos a ellos lo hacemos con la tentación de sacar ese algo a flote y con la intención de escribir sobre ese algo, o sobre otros algo, sin nunca acabar, siempre una nueva búsqueda, una nueva idea, un nuevo encuentro.

Así, en este artículo volvemos a Foucault, pero ahora queremos aproximarnos a él haciendo otras conexiones, otras búsquedas, pues sospechamos que sus ideas sobre la vida, el arte, la educación, la subjetividad, el pensamiento estético, etc. tienen otro algo para decirnos; asunto que nos motiva siempre a repensarlo, percibiendo una constante urgencia de movilizar el pensamiento. Así, asumimos a Foucault no como un guía del pensamiento, sino más bien como un filósofo que puso en crisis 
de forma explícita todos los mecanismos de transmisión del saber, lo asumimos como un antieducador o un contraeducador, "un maestro sin seguidores, su quehacer tritura, corta, disecciona, hurga y efectúa toda operación que tenga como móvil la convulsión activa de las cosas" (Esquivel, 2010, p. 4).

Lo que pretendemos es seguir los rastros de sensibilidad y educación en la textura de lo escrito por Michel Foucault, para intentar ver que podemos seguir tejiendo en lo que hemos venido construyendo en la Educación Corporal. No queremos confirmar lo que ya sabemos, sino, por el contrario, comenzamos a recorrer un camino entrecortado de luces y sombras, aprendiendo a pensar lejos de lo que ya sabemos para irnos acercando a lo que ignoramos pero sospechamos. Así, este acercamiento al pensamiento foucaultiano desde lo sensible y la educación se convierte en una escritura experiencial, en un devenir que potencia en nosotros el des-cubrir de algo que creemos podemos encontrar.

Ahora bien, la preocupación por el problema de una educación de lo sensible desde la perspectiva foucaultiana pasa también por la necesidad de ver la estrecha relación que guardan la educación y lo sensible con el arte. Así, por ejemplo, hemos encontrado el texto de Ricardo Arcos-Palma, Foucault y Deleuze: pensar lo sensible. Para una relectura con gafas para ciegos, donde el autor nos dice que el arte en general le sirvió de pre-texto a Foucault para elaborar su pensamiento filosófico: "Lo sensible abordado por Deleuze y Foucault parece tener una razón de ser, un camino lógico, pese a la aparente contradicción que se desprende entre pensar y sentir". Según Foucault, "efectivamente emitir un juicio verdadero o falso es un acto del pensamiento; este acto consiste en sentir que existe un vínculo, una relación... Pensar, como usted lo ve, es siempre sentir, y nada más que sentir" (ArcosPalma, 2006, p. 1).

Al mismo tiempo, nos llama la atención que Michel Foucault analiza las obras de Magritte (Esto no es una pipa) y de Velásquez (Las meninas) para evidenciar los límites del lenguaje y la representación; pues con ello podemos ver el pensamiento-artista propio de Foucault, que es un pensamiento creativo y atento a lo que el arte tiene para decirnos, e intuimos con ello que esto puede ser una forma de pensar lo sensible en la educación.

Nos indica Farina (2011) que hay algunos despliegues de lo sensible en el pensamiento filosófico de Foucault. Más específicamente, en el análisis de la formación de la experiencia estética del sujeto, a través de algunas nociones como la de experiencia, que dobla el pliegue subjetivo hacia dentro, y la de acontecimiento, que abre este pliegue hacia su afuera. Con el pensamiento sobre la formación del sujeto, en Foucault, podemos mirar también estas condiciones de sensibilidad leídas en clave pedagógica. 
Finalmente, como lo plantea Runge (2003, p. 217), nos interesa:

Un Foucault ya más pedagogo (teórico de la formación y de la subjetivación) -si así se me permite denominarlo- que debe ser visto, en palabras de Hoskins, como un criptoteórico de la educación, pues con el tiempo dejó ver, y cada vez con mayor claridad, los nexos y preferencias, aunque sutiles, que remitían sus ideas y cuestionamientos al campo pedagógico y educacional.

Podemos entonces darle una mirada pedagógica al tercer Foucault, incluso en Hermenéutica del sujeto, texto en el que se recogen los resúmenes de un curso dictado durante 1981 y 1982, no solo es susceptible de ser leído en clave pedagógica, sino que puede ser pensado como un gran aporte a la problemática de lo sensible en perspectiva educativa, tal como lo muestran Gómez y Castañeda (2011) en Foucault y el cuidado de sí: es un acto de re-interpretación y renovación de sí mismo.

Por ello vamos siguiendo los rastros de Foucault para pensar una educación de lo sensible, intentando hacer resonancia con su idea de hacer de la vida una obra de arte. Como decía el propio Deleuze, a propósito de Foucault, considerar la existencia como una obra de arte permite que el pensamiento-artista, que es creativo, invente posibilidades de vida, modos de existencia y nuevas maneras estéticas y éticas de vivir la vida (Arcos-Palma, 2006).

Para ello desarrollaremos la idea de lo sensible en Foucault en dos momentos: primero abordamos brevemente las teorías de algunos filósofos que hasta el momento se nos han hecho más sugerentes al tratar el problema de lo sensible desde lo gnoseológico, para así desembocar en lo que hemos comenzado a construir sobre educación de lo sensible. En el segundo momento de este escrito indagaremos en varias ideas del pensamiento foucaultiano que han hecho ruido en medio de dicha construcción.

\section{Educación de lo sensible}

Antes que nada, quisiéramos aclarar que lo que aquí se plasma son acercamientos conceptuales, sospechas, intuiciones y reflexiones que apenas están siendo construidas y configuradas en torno a lo sensible en perspectiva educativa, asunto que además parte de una inconformidad e incomodidad frente a esa visión de la educación, propia del platonismo, que se dirige hacia lo razonable y estrecha lo sensible. 
Tradicionalmente, la pretensión del conocimiento filosófico y científico ha vinculado el conocimiento con la verdad y con la necesidad de esta. La visión filosófica clásica del conocimiento comienza con Platón (428-347 a. C.), quien desarrolló una teoría según la cual las cosas particulares pueden entenderse en virtud de participar en ideas universales, inaugurando así el pensamiento metafísico occidental. Entre los años 395 y 370 a. C. escribe los considerados diálogos medios o de madurez, entre los que se encuentran La República, el Fedón y el Teeteto, los cuales exponen básicamente la teoría de las ideas y la naturaleza del saber. A su vez, el Hipias Mayor junto con el Fedro son considerados los diálogos estéticos de Platón y que tratan sobre lo bello.

En el Fedro, Platón explica el origen del ser humano. En este mito, el alma, que pertenece originalmente al mundo de las ideas, es como un carro tirado de dos caballos, uno manso y otro bravo, que están dirigidos por la razón. En el momento en que el alma cae y se encarna en un cuerpo olvida en cierto grado dichas ideas. Así, el conocimiento es el recuerdo de estas ideas y la materia es la sobra de estas ideas; dicha materia nos ofrece un estímulo que puede conducirnos a ellas, a través de un procedimiento que es el amor, de ahí que se desarrolle la idea de lo bello en relación con el amor y el conocimiento.
Platón propone la existencia de dos dimensiones en la realidad: el mundo inteligible y el mundo sensible. El mundo sensible es el que habitamos, en el que habita nuestro cuerpo, el mundo de los sentidos, un mundo transitorio. El mundo inteligible o de las ideas es el del verdadero conocimiento; para él las ideas puras no pueden ser percibidas meramente por los sentidos, pues el mundo de las ideas que son objetivas, eternas y universales es un mundo supraterrenal en el cual solo puede habitar el alma. No se llega a conocer las ideas despreciando los sentidos, sino que estos son usados, descubriendo lo que nos sugieren. Así hay una cooperación entre sentidos y razón en la marcha al conocimiento (Ross, 1986). Para Platón, las cosas sensibles están en constante flujo, aquello que percibimos no son sino sombras de una realidad, pura apariencia, y en cuanto apariencias muestran un mundo alejado de la verdad, de ahí que las cosas sensibles no deban ser objeto del conocimiento, sino que el conocimiento deba ser algo distinto de las cosas sensibles. De este modo, el acceso al verdadero conocimiento solo lo puede hacer el alma, pues al percibir las cosas únicamente a través de los sentidos hay una imagen engañosa de ellas, las sensaciones son defectuosas, puesto que solo el alma es capaz de reflexionar y cuestionar las cosas, elevándose en sí al conocimiento: 
(...) Si obtenemos un conocimiento suficiente de la unidad (...) por la vista o por cualquier otro sentido, este conocimiento no podría dirigirnos hacia la contemplación de la esencia (...) pero si la vista nos ofrece siempre en la unidad alguna contradicción (...) en este caso hay necesidad de un juez que decida; el alma, embaraza$\mathrm{da}$, despierta al entendimiento y se ve precisada a hacer indagaciones y a preguntarse a sí misma lo que es la unidad. El conocimiento de la unidad en este caso es una de las cosas que elevan el alma y la vuelve hacia la contemplación del ser (Platón, 2009, p. 282).

Para Platón, el alma tiene la facultad de conocer; esta facultad es dada por las ideas, pero en especial por la idea del bien que es el principio de la verdad. Es necesario elevar el alma al puro conocimiento y conducirla a la contemplación del ser, pero esto se logra a través de objetos que den origen a sensaciones contrarias invitando al alma a reflexionar, así, cuando los sentidos no se dan cuenta de que una cosa o la otra son opuestas, producen entonces solo sensaciones, y estas no llevan al espíritu a reflexionar, perdiéndose con ello de la posibilidad de acceder al conocimiento.

En el Teteto, Platón define que el conocimiento está constituido por creencias u opiniones verdaderas y justificadas. En este sentido, el conocimiento, además de restringirse a las creencias verdaderas y justificadas, debe también rechazar las sensaciones $y$, para afirmar que algo es conocimiento, sus razones deben ser bien fundadas, justificadas y suficientes, pues si no se estaría hablando de fe u opiniones, lo que él llamó el conocimiento Doxa, cuya formulación se basó en el conocimiento puramente sensible adquirido a través de los sentidos, que es también un conocimiento práctico, individual y subjetivo que toma lo real como aquello que es percibido y que no es estrictamente conocimiento.

Ahora bien, lo bello en Platón esta identificado con lo bueno. En El banquete se presenta la belleza como la manifestación superior del alma, esto es, que lo bello se alía con el bien y hasta se le subordina, a su vez que se alía con lo verdadero. Así, la belleza debe hallarse en el alma más que en el cuerpo; "un alma bella" esta más cerca al mundo de las ideas que un cuerpo hermoso. Sin embargo, la belleza reside en el mundo de lo sensible, en la apariencia, mientras que lo verdadero y lo bueno 
residen en el interior de las cosas. Pero, ¿cómo hacer para que lo bello resida esencialmente en las almas? Se requiere entonces un proceso intelectual en el que la idea es el escalón supremo de la dialéctica que conduce a dicho estado. La dialéctica empírica del amor transporta de plano en plano el alma enamorada, hasta llegar al plano mismo de las ideas. Así, lo bello se hace universal, al estar de lado de lo verdadero y de lo bueno (Bayer, 1965).

De esta manera, desde la teoría del conocimiento se instalan dos grandes perspectivas sobre las formas del conocer: el conocimiento intelectivo o la razón pura y el conocimiento sensible o la estética. Nociones que se situaron a lo largo de la historia en las teorías educativas, dando lugar a la separación entre lo sensible y el pensamiento, pero con una relevancia de lo intelectivo sobre lo sensible; ideas que dirigieron además el accionar educativo.

Ahora bien, en el campo de la filosofía, solo hasta 1750 podemos empezar a hablar de la sensibilidad como una teoría independiente de la razón. Autores como René Descartes en su obra Meditaciones metafísicas, Gottfried Leibniz con Discurso sobre la metafísica y Baruch de Spinoza en Ética demostrada según el orden geométrico, si bien problematizaron las formas de conocer y hablaron de los sentidos, la percepción y las afecciones, se quedaron en la idea de la razón y la abstracción como únicas y verdaderas formas del conocer. Pero en 1750, con Alexander Baumgarten (1714-1762), se le da a la estética el carácter de ciencia; en sus obras Reflexiones filosóficas acerca de la poesía (1735) y Aesthetica (1750), presenta la estética como teoría del conocimiento sensitivo. La Aesthetica es una noción que no solo trata acerca de los objetos bellos del arte y de la naturaleza, sino que comprende también una facultad de la percepción, a lo cual llamó "conocimiento sensible" (cognitio sensitiva), que es también conocimiento estético y que se diferencia del conocimiento conceptual y proposicional. Para Baumgarten, el conocimiento estético se concentra en la percepción de fenómenos complejos, más para hacer presente su densidad perceptual que para analizarlos en su composición. El conocimiento estético coloca su atención en lo particular; conoce lo particular en su particularidad, asunto que es inalcanzable para cualquier ciencia (Seel, 2010). Así, Baumgarten le da una independencia al conocimiento sensible, fundándolo como una forma de conocimiento y una corriente filosófica. Mientras que para Leibniz la intuición sensible era un "todavía no" del pensamiento, para Baumgarten era el análogo de la razón, con una función propia: representar el conjunto de la multiplicidad de los objetos sensibles (Soto, 1987). Para Baumgarten, el conocimiento sensible no es una forma más o 
menos imperfecta del conocimiento lógico, sino un modo de conocimiento propio, y aunque también lo denominó como un modo de conocimiento inferior, para él el conocimiento pleno se constituye mediante la conjunción del pensamiento de las ciencias y el pensamiento estético.

Continuamente, en 1787, el filósofo Immanuel Kant, en su primera parte de Crítica del juicio, refutaría a Baumgarten, argumentando que no puede considerarse la estética como un tipo inferior de la teoría del conocimiento. Kant insistió en que todas las fuerzas del conocer asisten a la percepción estética (Seel, 2010), colocando en un mismo nivel la sensibilidad y la razón, pero validando la sensibilidad en tanto esté acompañada de la razón y del entendimiento. La teoría kantiana consistirá en argumentar que hay dos modos de ser de la facultad del conocer. La primera es la receptividad, también llamada "facultad de la sensibilidad", la cual consiste en que el sujeto puede darse cuenta de la realidad de los objetos en el espacio y de la realidad de su propia conciencia en cuanto constante fluir de todo lo que puede representarse: los sentimientos, las sensaciones, los pensamientos, etc. La receptividad es el modo en el que el sujeto se da cuenta de que es afectado por los objetos que se le presentan a los sentidos (Carrillo, 2002). La segunda es la espontaneidad o facultad del pensar, que se refiere al poder del sujeto para producir por él mismo ciertas representaciones (conceptos) y que le permiten vincular y dar sentido a todas sus representaciones (ibíd.).

Kant quiso mostrar la necesaria conjunción entre la sensibilidad y el entendimiento según unas reglas. No basta con que una cosa meramente afecte los sentidos para que pueda constituirse en objeto de conocimiento, sino que además tiene que poder ser pensada a través de los conceptos. Así, ninguna de estas dos propiedades: sensibilidad y entendimiento han de preferir la una sobre la otra, pues ambas se concatenan entre sí: "Sin sensibilidad, no nos sería dado objeto alguno; y sin entendimiento, ninguno sería pensado. Pensamientos sin contenido son vacíos, intuiciones sin conceptos son ciegas" (Kant, 2006, p. 150). Sin embargo, para Kant tanto la sensibilidad como el entendimiento no pueden tampoco trocar sus funciones: "El entendimiento no puede intuir nada, y los sentidos no pueden pensar nada" (ibíd.). Es necesario distinguir y separar cuidadosamente unos de los otros, diferenciando la ciencia de las reglas de la sensibilidad, 
es decir, la estética, de la ciencia de las reglas del entendimiento, es decir, la lógica. Así llega Kant a la lógica trascendental, como ciencia del entendimiento puro que no puede pertenecer a la intuición y a la sensibilidad, además que los conceptos deben ser puros y no empíricos, es decir, el conocimiento que se presenta a nuestro espíritu es a priori. Se establece entonces nuevamente una defensa de la razón como única y exclusiva fuente de construcción del conocimiento, relegando la sensibilidad al dominio del intelecto.

Sin embargo, con el filósofo Friedrich Nietzsche la estética moderna toma un giro sustancial. En su obra El origen de la tragedia problematiza la estética desde la experiencia del arte, que "no implica un ascenso al espíritu objetivo o a las ideas puras, sino más bien el descenso a un 'ruido' libre de ideas" (Seel, 2010, p. 22).

La visión estética de Nietzsche tiene que ver con la relación que se teje entre la vida y el arte. Nietzsche encuentra en la Grecia Antigua formas de transgresión del sentir, del ser y del pensar el todo del Universo, lo que le inspira problematizar la vida bajo el estímulo del arte que permite vivir la vida misma. A través de las formas de vida, "apolíneas" y "dionisíacas", se puede acceder a un estado estético que encuentra placer en la indeterminabilidad. "No se trata pues de dominar la verdad, sino más bien se supera la creencia en la posibi- lidad y en el sentido mismo de una determinación absoluta de lo dado" (Seel, 2010, p. 23). El pensamiento de Nietzsche transgrede las macizas formas del conocimiento establecidas de la filosofía, ya no se trata de enumerar principios trascendentes, o de comprobar la existencia de Dios, ni de pensar con pretensiones de verdad; se trata más bien de soltar amarras, abandonar el puerto y partir hacia un devenir del pensamiento. Así, las formas de conocer desde la estética nietzscheana se inscriben ya no en búsquedas de verdades, ya no en dicotomías o dualismos sobre la razón o la sensibilidad, sino que se da una apertura a la sensibilidad misma. El racionalismo con vocación administrativa y previsora describe, conquista, trabaja, cifra el tiempo y el espacio y convierte al hombre y su destino en una ecuación de resultado irrefutable (Varela, 2010), pero la filosofía de Nietzsche ofrece frente a la verdad pequeña e incandescente que ofrece la ciencia otra verdad sublime que se levanta, que empieza a tejerse, que se configura como diversidad, como un mar opaco y turbulento, como un murmullo que se extiende y que solo el pensamiento estético está dispuesto a develar, a construir, a movilizar, a habitar. Podríamos atrevernos a decir que desde Nietzsche el asunto de la sensibilidad toma su efectiva independencia. De ahí que después de su pensamiento se expongan, ausculten, analicen, revisen y descarnen nuevos pensamientos acerca 
del arte y la vida como asuntos estéticos. Así también Nietzsche inspira el pensamiento de grandes filósofos contemporáneos como Gilles Deleuze, Jacques Derrida y, por supuesto, Michel Foucault.

De esta manera, nos adentramos entonces al concepto mismo de lo sensible. Para ello nos acompañaremos por un momento del pensamiento deleuziano acerca del concepto; podríamos comenzar diciendo que lo sensible hay que pensarlo bajo la noción de concepto propuesta por Gilles Deleuze en su obra Qué es la filosofía. Así, la construcción que se ha venido haciendo sobre lo sensible habita un estado de sobrevuelo respecto a sus componentes, apela a una construcción múltiple, plural y recíproca. Dicha construcción del concepto de lo sensible no quiere quedarse inmóvil, por el contrario busca siempre darle diferentes entradas, salidas, intensidades y movimientos a distintas nociones, teorías, formas, conceptos, ideas, sospechas y pensamientos que nos pongan en un lugar de encuentro, de descubrimientos, de creación. Acá lo sensible se piensa atravesado por un devenir; un devenir que, como diría Deleuze (1993, p. 22) "atañe en este caso a unos conceptos que se sitúan en el mismo plano. Aquí, los conceptos se concatenan unos a otros, se solapan mutuamente, coordinan sus perímetros, componen sus problemas respectivos, pertenecen a la misma filosofía, incluso cuando tienen historias diferentes." $Y$ se puede comprender que "incluso comprimidos, plegados y envueltos, estos elementos son potencias de ensanchamiento y de estiramiento del mundo" (Deleuze, 2008, p. 159). Cabría decir entonces que necesitamos crear un mundo, el mundo en el que sea posible una educación de lo sensible y, cuando se crea un concepto, se crea también otro mundo, un mundo de multiplicidad del pensamiento.

Así mismo es necesario aclarar que nombramos la sensibilidad como "lo sensible", porque nos alejamos de la visión empirista y clásica de la noción de sensibilidad que la concibe como aquellos datos que se nos son dados por medio de los sentidos. Acá también nos hemos valido del pensamiento deleuziano que plantea la sensibilidad como un problema trascendental. Esto se distingue de lo empírico en tanto que "lo trascendental remite a las condiciones mediante las que la experiencia nos es dada, condiciones que necesariamente no pueden ser sentidas en su uso empírico" (Galván, 2007, p. 231). Se plantea de esta manera el problema del "ser de lo sensible" que no puede ser re- 
ducido meramente a lo sentido, es decir, a la sensación empírica, sino que más bien nos envía a una instancia paradójica, pues lo sensible es aquello que es necesariamente sentido, pero que a su vez no puede ser sentido por los sentidos (Galván, 2007). Así, el ser paradójico de lo sensible es determinado en función de un "encuentro" que hace nacer la propia sensibilidad: "el objeto del encuentro (...) hace nacer realmente la sensibilidad en el sentido (...) no un ser sensible, sino un ser de lo sensible. No un dato, sino aquello por donde el dato se nos da. E igualmente resulta ser insensible de cierto modo" (Deleuze, citado por Galván, 2007, p. 232).

Así se plantea entonces una educación de lo sensible, no como una manera de potencializar, agudizar o formar las percepciones de los sentidos (lo que tocamos, olemos, oímos, entre otros), sino más bien como una actitud frente a lo que se nos da en el mundo. Lo sensible trata más bien sobre el pensamiento, sobre la actitud frente a la construcción de saberes, pero más que eso frente a la actitud de la construcción de la vida, una actitud que demanda constantemente un estado perceptivo frente a los signos del mundo.

En este sentido lo sensible tiene que ver con la actividad de la percepción, pero dicha actividad se extiende más allá de la simple percepción; no se trata de percibir algo meramente a través de los sentidos, el sujeto de lo sensible incluye necesariamente una atención capaz de conocer e interpretar. Se trata de una atención a lo indeterminable, de una percepción estética, de un estado estético. Nos dice Martin Seel (2010, p. 34) que "la atención estética dirigida a un acontecimiento del mundo externo es (...) una atención destinada a nosotros mismos: atención al instante aquí y ahora". Así la actividad de lo sensible tiene qué ver con cómo uno aprende a dar acogida y sentido a lo que advierte (Farina, 2005), significa la irrupción de la indeterminación, como un acontecimiento sobre lo que es determinado, como una forma de afección que atiende a la presencia de posibilidades desconocidas, habita la incertidumbre, el devenir, está atento a las cosas próximas, hace referencia a estados de pensamiento que producen conocimientos móviles que nunca se detienen, esto es, un constante transcurrir por nuevos escenarios, nuevas formas de ser, hacer y de pensar apela a una atención que entiende que la realidad es más rica que todo cuanto puede ser conocido en ella solo mediante el conocimiento proposicional, haciendo visible una frontera de toda comprensión del mundo, ante la cual ninguna teoría del conocimiento puede ser ciega (Seel, 2010). La percepción que pertenece a lo sensible es una percepción que conmueve el ser, que envuelve, que es constante, abierta y despierta. 


\section{Tras las huellas del pensamiento foucaultiano en perspectiva de lo sensible}

Ahora bien, ir tras los rastros de Foucault en la sospecha de lo que de él podemos empezar a configurar como una educación de lo sensible lleva a descubrir que no solo sus rastros pedagógicos se nos prestan propicios para dicha construcción, sino también la estrecha relación de su pensamiento y filosofía con la vida problematizada desde el arte, el cuidado de sí, la formación del otro, la literatura, la cartografía, entre otros.

De este modo comenzamos abordando el arte y la literatura como parte de su pensamiento filosófico. Un ejemplo de ello nos lo presenta Farina (2011) al decir que Foucault coincidió con la brasileña Lygia Clark en el París de finales de los 60 y de los primeros años de los 70. Coincidieron, también, en la transgresión de toda una racionalidad asentada sobre el plano del pensamiento dominante de Occidente, del sujeto de la experiencia de esa racionalidad, y del cuerpo de ese sujeto, hecho a la medida del saber. Coincidieron en las relaciones que sostenían la "forma-sujeto", y en la percepción del mundo que configuraba esa perspectiva. Lygia Clark, en el terreno estético, y Foucault, en el campo discursivo, se enfrentaban a los modos en que el conocimiento del mundo por parte del sujeto, había instituido un falso equilibrio asentado en el plano de la racionalidad, que perennizaba sus propias formas y saberes. La estética de Lygia Clark se afirma como expresión de una nueva política de lo sensible para una ética como creación de las formas de la existencia.

Otro ejemplo de ello, y como se mencionó en la introducción, Arcos-Palma (2006) nos plantea que cuando Foucault lee a Artaud, Borges, Velásquez, Magritte, entre otros, le sirve de pretexto para comprender el problema de la representación y el enunciado dentro del campo del lenguaje, donde lo que se nombra hace visible aquello que permanece oculto. Es importante tener en cuenta que ese acto nominativo propio del lenguaje da cuenta de la estrecha relación entre las palabras y las cosas. Precisamente, este problema lo desarrolla más hondamente en su texto Las palabras y las cosas. Llama la atención que en las primeras líneas del prefacio de este libro se encuentren frases como: "Este texto nació de un texto de Borges. De la risa que sacude, al leerlo, todo lo familiar al pensamiento-al nuestro: 
al que tiene nuestra edad y nuestra geografía, trastornando todas las superficies ordenadas y todos los planos que ajustan la abundancia de seres provocando una larga vacilación e inquietud en nuestra práctica milenaria de lo Mismo y lo Otro" (Foucault, 2001a, p. 1). En el mismo texto se resalta el análisis que hace de la obra Las meninas, de Velásquez, en torno al espectador:

El pintor está ligeramente alejado del cuadro. Lanza una mirada sobre el modelo; quizá se trata de añadir un último toque, pero también puede ser que no se haya dado aún la primera pincelada... el pintor contempla, el rostro ligeramente vuelto y la cabeza inclinada hacia el hombro. Fija un punto invisible, pero que nosotros, los espectadores, no podemos asignar fácilmente, ya que este punto somos nosotros mismos: nuestro cuerpo, nuestro rostro, nuestros ojos (Foucault, 2001a, p. 13ss).

Por otro lado, leyendo este filósofo en perspectiva pedagógica, Runge ha dejado señalado que Foucault tiene que ver con dos problemas fundamentales que han sido típicos en la pedagogía o ciencia de la educación, a saber: el problema de la formación humana y el problema de la relación pedagógica que se da entre maestro y discípulo. Agrega que:

Quien tenga un conocimiento general de las teorías clásicas de la formación (...) y de los planteamientos de la pedagogía de las ciencias del espíritu (...) desarrollados en el ámbito alemán a comienzos del siglo pasado, estará de acuerdo conmigo en no considerar descabellado el anterior etiquetado que hago del libro Hermenéutica del sujeto de Foucault (...) Uno podría decir que los últimos trabajos de este autor que se refieren a los procesos -prácticas y técnicas- de subjetivación se pueden ver, ante todo, como grandes aportes a una teoría general de la formación (Runge, 2003, p. 3).

\section{"Ocúpate de ti mismo"}

El precepto ocuparse de sí o cuidado de sí era uno de los más grandes principios de la antigüedad, una de las más importantes reglas de la vida social y personal y uno de los fundamentos del arte de vivir, una práctica constante y no simplemente una actitud. En los textos Tecnologías del yo y Hermenéutica del sujeto, que están enteramente dedi- 
cados al estudio de la cultura del sí mismo, desde el momento socrático-platónico y hasta la filosofía helenístico-romana, pasando por el cristianismo, Foucault nos revela su inquietud por indagar sobre la historia de las diferentes maneras en que, en la cultura, los seres humanos han hecho y han ampliado un saber acerca de sí mismos, desarrollando de esta manera una hermenéutica del yo en un contexto histórico que aún hoy se presenta como un principio délfico y que nos inquieta a movilizar nuestro pensamiento.

Este estudio del principio délfico que nos hace Foucault, desde una perspectiva de la formación, presupone entonces que en el ser "algo pasa", que aquel que se preocupa de sí como un principio de subjetivación es quizás un sujeto de lo sensible: inquieto por la formación como una posibilidad de llegar a ser otro a través de la búsqueda de la verdad, pero no de una verdad del saber solamente, sino también del ser, del saber ser, del hacer y de habitar de otra forma el mundo. En este sentido, nos dice Farina (2005, p. 43.) que:

Tratar de vivir una verdad tiene más que ver con los pactos y elecciones que uno hace consigo mismo y en relación con los demás, que con la adhesión a órdenes morales. El cuidado de la verdad expone al juego discursivo un repertorio de saberes, a través de los cuales se da sentido a los modos de vida, a las formas de la conciencia y a una determinada actitud.

\section{"La formación humana"}

Para Foucault, el imperativo "ocuparse de sí" gira en torno a lo que se podría llamar la cultura de sí, la formación de sí, la SeIbstbildung, la autoformación. A lo largo de su escritura, devela cómo el ocuparse de sí implica una actitud de autoformación, la preocupación por uno mismo se traduce en el acceso a prácticas y actitudes que demandan una transformación de su propio ser. La historia del cuidado y de las técnicas de sí son, entonces, una forma de hacer la historia de la subjetividad, pero a través de la formación y transformación de la relación consigo mismo. La preparación del individuo tiene esencialmente ligado el aspecto formativo en tanto que no se trata de una preparación solo para la profesión o una actividad social, sino que se trata de formar al individuo para que pueda soportar como es debido todos los 
acontecimientos negativos o positivos que puedan venir.

Sin embargo, es nuestro interés problematizar desde una educación de lo sensible el cuidado de sí como un asunto que debe comenzar en el maestro, en el formador, en el docente, así entonces, si vamos tras la ruta de lo sensible, no sería entonces coherente pensar una educación de lo sensible en donde esté ausente un maestro de lo sensible. En la relación maestro-discípulo que aborda Foucault en su obra Hermenéutica del sujeto se plantea que en el modelo helenístico se hace especial énfasis en el cuidado de sí como requisito fundamental para el cuidado de los otros. Siguiendo a Runge (2003), la incapacidad de cuidar de sí se traducía en incapacidad para cuidar de los otros (gobernar-educar). Era necesario saber tratarse a sí mismo y saber estar consigo mismo, para poder saber tratar a los otros y saber estar en sociedad. Pero este modelo también enfoca su mirada en la ignorancia no solo de las cosas que deben saberse, sino también en la ignorancia de sí mismo. "El cuidado de sí es ético en sí mismo; pero implica relaciones complejas con los otros, en la medida en que este éthos de la libertad es también una manera de ocuparse de los otros" (Foucault, 2002, p. 399). Quien se ocupa de sí mismo aprende a ser capaz de ocupar un lugar adecuado en su comunidad, en su ciudad $o$ en general en sus relaciones interindividuales. Una educación de lo sensible requiere que el maestro vivencie experiencias estéticas que supongan una violación de la continuidad de esos modos que abraza lo inesperado, que se afirma en lo colectivo, de manera relacional y afirmando una composición inédita (Farina, 2005).

Para Foucault (2002, p. 73), "el maestro es el principio y el modelo del cuidado de uno mismo que el joven debe tener de sí en cuanto que sujeto", de ahí la necesidad de pensar en un maestro de lo sensible como dispositivo para la construcción de una educación de lo sensible; un maestro que primero sea capaz de ocuparse de sí, de inquietarse de sí, para luego poder ser guía que, a su vez, genere condiciones de sensibilidad que movilicen la formación de sus discípulos.

$Y$ es que "no es posible educar a los otros, no se les puede educar bien, no es posible transformar los propios privilegios en acción educativa sobre los otros, en acción racional, si uno se ha preocupado por sí mismo" (Foucault, 2002, p. 51).

Stephen Riggins, en una entrevista que realizó a Foucault en 1983, le pregunta: "¿Hay una especial afinidad en su estilo de filosofar y las artes en general?", a lo que Foucault contesta:
Mi trabajo intelectual está relacionado con lo que usted podría llamar esteticismo, que signifi- ca transformado por uno mismo (...) No estoy in- teresado en el estatus 
académico que he alcanzado porque mi problema es mi propia transformación (...) también el porqué, cuando la gente dice, "bien, usted pensó esto unos pocos años atrás y ahora usted está diciendo otra cosa", mi respuesta es, "bien, ¿usted piensa que he trabajado como todos estos años para decir el mismo pensamiento y no ser cambiado? Esta transformación de uno mismo por su propio conocimiento es, pienso, una cosa más bien cerrada para la experiencia estética. Porque ¿podría trabajar un pintor si él no es transformado por su propia pintura?

El arte fue un pre-texto en el pensamiento de Foucault, un pre-texto para pensar lo sensible. De esta manera, podríamos decir que si el cuidado de sí es un estado estético de la vida, el arte es entonces una práctica estética que tiene que ver con una percepción que irrumpe en el sujeto; pero no solo es una percepción que irrumpe para movilizar el pensamiento, sino que se convierte también en un principio de la vida misma: así, el cuidado de sí en relación con el arte permitirá pensar la vida y el arte bajo una misma textualidad. Como nos indica Farina (2005, p. 51), "el sujeto de una vida como obra de arte cuida de la parcela reflexiva en la que él mismo se constituye como tarea ética". Es decir, un maestro de lo sensible, en la experiencia de pensar sobre el campo del arte, traza líneas de vida, que constituyen su vida misma.

Pensar la relación arte y vida es pensar en un estado estético que se deja afectar a través de prácticas de sí, en las que lo importante no es aprender un cuerpo de conocimientos y contenidos sistemáticos de un saber, sino la elaboración y reelaboración de formas de relación reflexiva sobre sí mismo a través del arte.

Por su parte, el concepto de ética como estética de la existencia que mana de los estudios de Foucault sobre los procesos de subjetivación es uno de los más potentes y sugestivos para una educación de lo sensible. Nos dicen Gómez y Castañeda (2011) que para Foucault la vida misma de cada sujeto es una obra de arte que día a día se construye, se moldea, se recrea, se imagina y se proyecta, constituyéndose en la propia esencia estética y artística de cada uno, en la que piensa lo que dice y dice lo que piensa acerca de sus propias experiencias, ya que uno es "sujeto de cierta cantidad de cosas: sujeto de acción instrumental, sujeto de relaciones con el otro, sujeto de comportamientos y sujeto 
de relaciones consigo mismo" (Foucault, 2002, p. 71).

Una forma de existencia puede ser considerada como una experiencia estética; esta se entiende como el proceso de configuración de la subjetividad, la forma en la que el sujeto se apropia de su propia experiencia, es el arte de vivir. En Foucault, el arte de vivir es coextenso con la inquietud de sí y con la vida individual y se ubica en el momento en que los jóvenes dejan de estar en manos de los pedagogos y entran en la vida con el fin de ejercer en ella un poder activo. La referencia histórica fundamental propuesta por Foucault para la expresión del arte de vivir es el Alcibíades de Platón, diálogo en el que se privilegia la inquietud de sí como necesaria para la existencia humana. Este arte de vivir se da en un momento preciso y oportuno: en griego, un kairos. Desde el momento de la adultez, la inquietud de sí se convierte en un imperativo categórico.

El maestro de lo sensible es entonces un maestro filósofo, un maestro artista, que percibe y articula los signos del arte provocando una variación en lo que se ve y se dice sobre lo real y que produce una especie de salto, de movimiento brusco en el pensamiento y en la vida, no busca una educación que solo construya y transmita experiencias objetivas del mundo exterior, sino que más bien construye y transmite las experiencias sobre sí mismo, como otra forma de educación.
Finalmente, un maestro de lo sensible es como un texto escrito desde las huellas de su experiencia, condensando potencias que pueden ser reactivadas en la lectura de su vida, su vida como obra de arte.

\section{Referencias}

Arcos-Palma, R. (2006). Foucault Deleuze: pensar lo sensible: para una relectura con gafas para ciegos. Ponencia Simposio de Estoica. I Congreso Colombiano de Filosofía. Bogotá.

Baumgarten, A. (1955). Reflexiones filosóficas acerca de la poesía. Buenos Aires: Aguilar.

Bayer, R. (1965). Historia de la estética. México: Fondo de cultura económica.

Carrillo, L. (2002). Tiempo y mundo de lo estético. Sobre los conceptos kantianos de mundo, tiempo, belleza y arte. MedeIlín: Editorial Universidad de Antioquia.

Deleuze, G. (1986). Foucault. Barcelona: Paidós.

Deleuze, G. \& Guattari, F. (1993) ¿Qué es la filosofía?. Barcelona: Anagrama.

Deleuze, G. \& Guattari, F. (2008). Mil mesetas. Capitalismo y esquizofrenia. Valencia: Pre-Textos.

Descartes, R. (1990). Meditaciones metafísicas. Medellín: Edilux.

Esquivel, S. (2010). Michel Foucault educador (sobre la educación y el pensamiento contemporáneo). Ponencia X Congreso Nacional de Investigación Educativa.

Veracruz. 
Farina, C. (2005). Arte, cuerpo y subjetividad. Estética de la formación y Pedagogía de las afecciones. (Tesis doctoral). Universidad de Barcelona.

Farina, C. (2011). Arte, cuerpo y producción de sí. Texto inédito.

Foucault, M. (1981). Esto no es una pipa (ensayo sobre Magritte). Barcelona: Anagrama.

Foucault, M. (1990). Tecnologías del yo y otros textos afines. Barcelona: Paidós.

Foucault, M. (1999). Estética, ética y hermenéutica: Obras esenciales. Volumen III. Barcelona: Paidós.

Foucault, M. (2001a). Las palabras y las cosas. Buenos Aires: Siglo XXI.

Foucault, M. (2001b). Historia de la sexualidad. El uso de los placeres. México: Siglo XXI.

Foucault, M. (2002). La hermenéutica del sujeto. México: Fondo de Cultura Económica.

Foucault, M. (2005). Historia de la sexualidad. La inquietud de sí. Madrid: Siglo XXI.

Foucault, M. (2009). El gobierno de sí y de los otros. Buenos Aires: Fondo de Cultura Económica.

Gallo, L. E. \& García, C. (2011). Epílogo: La educación corporal en perspectiva pedagógica. En Gallo, L. E. (Ed.). Aproximaciones pedagógicas al estudio de la educación corporal. Medellín: Funámbulos Editores, Universidad de Antioquia, pp. 291-302.

Galván, G. (2007). Gilles Deleuze: Ontología, pensamiento y lenguaje. Un logos problemático. Granada: Universidad de Granada.

Gómez, S. N. \& Castañeda, G. (2011). Foucault y el cuidado de sí: un acto de re-interpretación y re-novación de sí mismo. En Gallo, L. E. (Ed.). Aproximaciones pedagógicas al estudio de la educación corporal. Medellín: Funámbulos Editores, Universidad de Antioquia, pp. 105-143.

Kant, I. (2006). Crítica de la razón pura. Madrid: Tecnos.

Leibniz, G. (1962). Discurso de metafísica. Madrid: Aguilar

Montoya, J. A. (2011). El cuerpo en perspectiva de una subjetivación política. Un ámbito de estudio de la Educación Corporal. Educación Física y Deporte, 30(2), pp. 571-577.

Mora, J. (2002). Diccionario de Filosofía. Barcelona: Ariel.

Platón. (2009). La República. Bogotá: Panamericana.

Ross, D. (1986). Teoría de las Ideas de Platón (Traducción de José Luis Díez). Madrid: Cátedra. 
Runge, A. (2003). Foucault o de la revaloración del maestro como condición de la relación pedagógica y como modelo de formación. Una mirada pedagógica a la hermenéutica del sujeto. Revista Educación y Pedagogía, 15(37), pp. 217-232.

Seel, M. (2010). Estética del aparecer. Buenos Aires: Katz.

Soto, M. J. (1987). La Aesthetica de Baumgarten y sus antecedentes leibnicianos. Anuario Filosófico, 20(2). Recuperado de http://dspace.unav.es/dspace/ bitstream/10171/2298/1/08.\%20 M A R \% C $3 \% 8$ D A \% 20 JES \% C 3 \% 9AS \% 20SOTO \% 20 B R U N A , \% $20 \mathrm{~L}$ a \% 20 $\%$ C2\%ABaesthetica\%C2\%BB\%20 de $\% 20$ Baumgarten $\% 20 y \% 20$ sus $\% 20$ antecedentes $\% 20$ leibnicianos.pdf.

Spinoza, B. (1987). Ética demostrada según el orden geométrico. Madrid: Alianza Editorial.

Varela, G. (2010). Nietzsche. Una introducción. Buenos Aires: Quadrata. 